\title{
Bile Pigment Composition and Bilirubin Esterification in the Developing Chick
}

\author{
PIETRO VAJRO ${ }^{1}$ M. MICHAEL THALER, AND NORBERT BLANCKAERT \\ Departments of Pediatrics [P.V., M.M.T.], Laboratory Medicine [N.B.] and Liver Center University of \\ California School of Medicine, San Francisco, California and Laboratory of Biological Chemistry, \\ Department of Molecular Cell Biology, Catholic University of Leuven, Leuven, Belgium [N.B.]
}

ABSTRAC'T

\begin{abstract}
In vivo and in vitro bile pigment metabolism was studied in embryos, chicks, and mature White Leghorn hens. Biliverdin and bilirubin pigments were present in bile of embryos from the earliest stage examined $(14 \mathrm{~d})$. Bilirubin accounted for 2.5$11.5 \%$ of total bile pigments, with the higher percentages in the early embryo. Biliverdin was exclusively in unesterified form. In contrast, bilirubin was almost entirely $(>98 \%)$ in esterified form. Glucosides consistently predominated over glucuronides and xylosides $(6: 3: 1)$. In serum, bilirubin and biliverdin were undetectable at all embryonic stages and after hatching. Bilirubin UDP-glycosyltransferase activities with UDP-glucuronic acid, UDP-glucose, and UDP-xylose were detectable in chick embryo liver and averaged 67,72 , and $102 \%$, respectively, of the corresponding adult mean values, without significant change throughout development. We conclude that multiple bilirubin esterification systems mature early in the avian embryo. This is in marked contrast to the development of bilirubin metabolism in the mam-
\end{abstract}

In mammals, metabolism of numerous xenobiotics and endogenous compounds (e.g. bilirubin) occurs in the liver and is catalyzed by a family of microsomal UDP-glucuronate $\beta$-glucuronosyltransferase (acceptor nonspecific), EC 2.4.1.17 (1) isoenzymes, primarily with UDPGlcUA as sugar donor cosubstrate (2). A number of these isoenzymes have been purified and the primary cDNA sequence of several glucuronyltransferases has been elucidated $(3,4)$. These individual isoenzymes display substrate selectivity, are differently affected by classical microsomal enzyme-inducing agents such as phenobarbital, 3-methylcholantrene, and clofibrate, and exhibit a different pattern of development in the fetal and neonatal periods $(2,5)$.

Glycosylation of xenobiotics and endogenous metabolites is often markedly lower in the fetus compared with adults. Inac-

Received December 2, 1994; accepted April 5, 1995.

Correspondence and reprint requests: Norbert Blanckaert, M.D., PhD., Department of Clinical Pathology/UZL Medical Center, Capucynenvoer 33, B-3000 Leuven, Belgium. Supported by grants AM-11275, AM-26743, and AM-07179 from the National Instilutes of Health and by grants from the Belgian National Lottery, Belgian National Bank, and Belgian National Research Council. P.V. was a recipient of a North Atlantic Treaty Organisation Research Fellowship.

L Present address: Department of Pediatrics, University of Naples, 1-80131, Naples, Italy. malian fetus in which detoxication of this potentially toxic pigment is achieved by placental clearance, and bilirubin esterification matures only after delivery. These contrasting developmental patterns are consistent with an adaptive response to the different requirements in the avian embryo and mammalian fetus for self-protective detoxification of unconjugated bilirubin. (Pediatr Res 38: 349-355, 1995)

\author{
Abbreviations \\ UDPGIcUA, UDP-glucuronic acid \\ UDPGlc, UDP-glucose \\ UDPXyl, UDP-xylose \\ Chapso, 3-(-cholamido-propyl)dimethylammonio-2-hydroxy-1- \\ propane sulfonate
}

Hepes, $\mathrm{N}$-2-hydroxyethylpiperazine- $\mathrm{N}^{\prime}$-2-ethanesulfonic acid Azpm, azopyrromethene

tivation and safe elimination of potentially toxic compounds produced by the mammalian fetus largely depends on transfer via the placenta and disposal by the mother. For several UDP-glucuronyltransferase isoenzymes, hepatic conjugation matures only after birth so that conjugation deficiency occurs in the neonatal period, resulting in transient accumulation of potentially toxic metabolites in the body (6). A clinically important and obvious example of this situation is neonatal hyperbilirubinemia, which is due to accumulation of unconjugated bilirubin in tissues and body fluids and occurs in most mammals including humans. Effective disposal of bilirubin requires conversion to bilirubin glycosides. Severe unconjugated hyperbilirubinemia may produce irreversible neurologic damage. Therefore, induction of bilirubin esterification in the fetus and newborn is of therapeutic importance.

Investigations on induction and development of in vivo conjugation are complicated in mammalian test models by interference of maternal factors and by difficult access to the fetus. To circumvent these difficulties, several investigators have used the embryonated egg as a model system $(2,7)$. Hepatic UDP-glucuronyltransferase activity toward various 
phenolic substrates increases from virtually undetectable to adult values in chick embryo liver and kidney on hatching at 21 d (8). Relatively few results are available on bilirubin esterification in the avian embryo. There is circumstantial evidence for developmental changes in bilirubin esterification similar to the pattern found in mammals. Transient hyperbilirubinemia in the posthatching period (9) and absence of bilirubin UDPglucuronyltransferase activity in chick liver microsomes (10) have been reported. On the other hand, there is evidence for in vivo and in vitro formation of bilirubin glucosides, xylosides, and glucuronides by adult chickens (11-13). These data and the findings that precocious development in vivo of UDPglucuronyltransferase can be elicited and marked enzyme induction be obtained by injection of phenobarbital or infusion of certain hormones into embryonated eggs $(8,14)$ prompted us to evaluate the developing chick embryo as a model system for studies on ontogenic/adaptive aspects of bile pigment metabolism and modalities for treatment of unconjugated hyperbilirubinemia.

A major deficiency of the previous studies on development of bile pigment metabolism $(9,13,15)$ in avian species is that nonspecific and insensitive analytical tools were used for determination of in vivo and in vitro esterification of the pigments. Therefore, it was necessary to reinvestigate the composition of bile pigments in serum and bile and the bilirubin UDP-glycosyltransferase activities in the embryonic chick liver throughout its development using advanced methodology for identification of bile pigments and assay of enzymic esterification of bilirubin $(16,17)$.

\section{METHODS}

Chemicals and test pigments. All solvents and reagents were analytical grade. Bilirubin, UDPGlcUA, UDPGlc, UDPXyl, bovine serum albumin, Cohn fraction V, Hepes, $\mathrm{NAD}^{+}$, and glucaro-1,4-lactone were from Sigma Chemical Co. (St. Louis, MO). Chapso was obtained from Bio-Rad Laboratories (Richmond, CA). Ethyl anthranilate and pentan-2-one were from Eastman Kodak Co. (Rochester, NY). HPLC-grade chloroform (pentene stabilized, or $0.75 \% \mathrm{C}_{2} \mathrm{H}_{5} \mathrm{OH}$ preserved) was from Fisher Scientific (Fair Lawn, NJ). Glass plates precoated with silica gel (5763/0025 from Merck, Darmstadt, Germany) were used for thin-layer chromatography.

The following reference compounds were prepared as reported previously: bilirubin C-8 monomethyl ester, bilirubin C-12 monomethyl ester, bilirubin dimethyl ester, and xanthobilirubic acid monomethyl ester (18); bilirubin-IX $\beta, I X \gamma$, and $-\mathrm{IX} \delta$ in their unesterified form, monomethyl ester form, and dimethyl ester form (19); bilirubin-III $\alpha$ and -XIII $\alpha$ (20); and the dimethyl esters of biliverdin-IX $\alpha,-\operatorname{IX} \beta,-\mathrm{IX} \gamma$, and IX $\delta$ (18). The monomethyl ester and unesterified forms of the latter four verdin isomers were prepared by partial saponification (18), and were isolated by thin-layer chromatography using chloroform/methanol/water (40:9:1, vol/vol) as solvent system. Isolated pigments were eluted from silica with methanol. In agreement with the recommendations of the IUPAC/IUB Commission on Biochemical Nomenclature, the names bilirubin and biliverdin represent, respectively, bilirubin-IX $\alpha$ and biliverdin-IX $\alpha$ (1).

Animals and collection of serum, bile, and liver tissue specimens. White Leghorn chickens (4-7 mo of age), chicks, and fertilized eggs were used. Body fluids and liver tissue specimens were collected at 14 and $18 \mathrm{~d}$ of embryonal age, immediately after hatching ("d 0"), and 1, 3, 4, 5, 7, 10, 14, and $18 \mathrm{~d}$ after hatching, and from adult animals. Chicks and chickens were fasted overnight before collection of samples. Blood for preparation of serum was obtained from adult animals by brachial venipuncture, from chicks by heart puncture, and from embryos by venisection of a chorioallantoic blood vessel.

Individual gallbladder bile specimens were obtained from adult animals at laparotomy, by direct puncture and aspiration of bile. In embryos and chicks, the gallbladder was removed after ligature of the ductus cysticus, and the contents of five to seven gallbladders from animals identical in age were pooled. After collection of blood and bile, liver tissue was excised, weighed, and minced with a razor blade. Liver homogenate $(25 \%, \mathrm{wt} / \mathrm{vol})$ was prepared in ice-cold $0.25 \mathrm{M}$ sucrose containing $1 \mathrm{mM}$ EDTA in $5 \mathrm{mM}$ Tris/HCl buffer, pH 7.4.

Rat bile enriched with added unconjugated bilirubin (18) was used as a source of reference pigments for HPLC analysis of bilirubins (see below). Azopyrromethenes prepared by treatment of dog gallbladder bile with diazotized $O$-ethyl anthranilate were employed as reference material for structural analysis of bilirubins in the avian body fluids (19).

Determination and structural analysis of bilirubins. Specific measurement of unesterified bilirubin and its di-esterified and two isomeric (C-8 and C-12) mono-esterified sugar conjugates was done with the alkaline methanolysis procedure combined with normal-phase HPLC (20). For each developmental stage, the identity of the bilirubin ester peaks, in at least one chromatogram, was verified by taking a duplicate sample through the alkaline methanolysis procedure, except that methanol replaced the $\mathrm{KOH} / \mathrm{methanol}$ reagent. In this "sample blank," transesterification does not occur, and peaks corresponding to methyl esters derived from sugar conjugates do not appear in the chromatogram. Because an unknown pigment partially co-eluted (slightly ahead) of unesterified bilirubin in most chromatograms from avian bile, accurate measurement of this minor bilirubin fraction was impossible. This interference led to overestimation of the unconjugated pigment and hence of total bilirubin values. However, the latter error was negligeable ( $<2 \%$ error for total bilirubin values) because the unconjugated bilirubin fraction, even when overestimated and uncorrected, invariably accounted for less than $2 \%$ of the total bilirubins in the chromatograms.

Bilirubins in the avian specimens were converted to ethyl anthranilate azoderivatives to identify the nature of the conjugating sugar moieties in the esterified bilirubins and to detect $\beta, \gamma$, and $\delta$ isomers of bilirubin-IX (21). Chemical and chromatographic methods for preparation and analysis of these azopigments and nomenclature used to denote the various azopyrromethenes are as previously reported (19). The method of Novros et al. (22) was used for measurement of total bilirubins by a diazo-method. 
Determination and structural analysis of biliverdins. Biliverdin in serum and bile was assayed by HPLC with a Whatman Partisil $5 \mathrm{CCS} / \mathrm{C}_{8}$ column (23). This method also permits separation of the four isomers $(\alpha, \beta, \gamma, \delta)$ of biliverdin-IX. The four isomers were used as chromatographic references, and the assay was calibrated with standards of biliverdin dissolved in methanol. Results of the chromatographic method were within $10 \%$ of the values found with the standard spectrophotometric method (24) when the two assays were applied in parallel to 10 different chicken bile specimens. The alkaline methanolysis procedure (18) was used to test for the presence of sugar conjugates of verdins in bile. Biliverdin monomethyl esters and biliverdin dimethyl ester were searched for in the reaction mixture by thin-layer chromatography with chloroform/ methanol/water (40:9:1; vol/vol) as solvent system, using isolated monomethyl esters and dimethyl esters of biliverdin-IX $\alpha$, IX $\beta$, -IX $\gamma$, and -IX $\delta$, and the unesterified form of the four isomers as chromatographic references.

Assay of bilirubin UDP-glycosyltransferase activities. Enzymic formation of esterified bilirubins was assayed with the $O$-ethyl anthranilate method (17) as described elsewhere (25). To ensure measurement of total transferase activities, homogenate was pretreated with the detergent Chapso to fully disrupt the microsomal vesicles (26). Preliminary experiments showed that activation of bilirubin UDP-glucuronyltransferase in hen homogenate was 7-fold and 6-fold when, respectively, Chapso and digitonin (17) were used as detergent. Incubation mixtures (total volume: $2.0 \mathrm{ml}$ ) consisted of the following components (final concentrations are in parentheses): $500 \mu \mathrm{l}$ of a $25 \%$ liver homogenate $(7.5-12.5 \mathrm{mg}$ of protein $/ \mathrm{ml}$ of total incubation mixture), Hepes buffer ( $\mathrm{pH} 7.7,140 \mathrm{mM}), \mathrm{MgCl} 2$ $(6.25 \mathrm{mM}), \mathrm{NAD}^{+}(10 \mathrm{mM})$, glucaro-1,4-lactone $(1 \mathrm{mM})$, Chapso $(4 \mathrm{mM})$, bilirubin and albumin $(100$ and $50 \mu \mathrm{M}$, respectively), and UDP-sugar cosubstrate $(5 \mathrm{mM})$. Bilirubin was dissolved in $0.05 \mathrm{M} \mathrm{NaOH}$ containing $1 \mathrm{mM}$ EDTA and then mixed with albumin dissolved in water. Except for UDPsugar, all components of the incubation mixture were combined at $0^{\circ} \mathrm{C}$, and the solution was blanketed with an argon atmosphere. For thermal equilibration, the mixture was preincubated for 5 min in a shaking water bath at $37^{\circ} \mathrm{C}$. The glycosidation reaction was started by addition of UDP-sugar dissolved in Hepes buffer $(100 \mu \mathrm{l}$ for each $2.0 \mathrm{ml}$ of total incubation mixture) and incubation at $37^{\circ} \mathrm{C}$ was continued for up to $20 \mathrm{~min}$. Aliquots $(500 \mu \mathrm{l})$ were withdrawn from the incubation mixture 5, 10, and 20 min after adding cosubstrate. Each portion was transferred to $1 \mathrm{ml}$ of ice-cold glycine- $\mathrm{HCl}$ buffer (0.4 M HCl; pH 2.4) and kept in melting ice. Coupling of esterified bilirubins was performed at $25^{\circ} \mathrm{C}$ as described elsewhere (17). Product formation progress curves were linear for 20 min under the selected incubation conditions. Blanks prepared by replacement of the cosubstrate solution by Hepes buffer were not used because esterified bilirubins (predominantly bilirubin glucosides) were formed during the pre-assay thermal equilibration period. Sufficient endogenous UDP-sugar appeared to be present in avian liver homogenate to produce significant esterification in the absence of added cosubstrate. Enzymic activities are expressed as pmoles esterified bilirubin/ $\mathrm{min} / \mathrm{mg}$ of protein.

At each tested developmental stage, verification of the nature of the enzymically formed esterified bilirubins was performed by structural analysis of the azoderivatives extracted from the incubation mixture. The underivatized azopyrromethenes were subjected to thin-layer chromatography for isolation of the $\alpha_{2}, \alpha_{3}$, and $\delta$ azopigment fractions. The identity of these bands (respectively, xyloside, glucoside, glucuronide) was confirmed by thin-layer chromatography of the acetylated (for $\alpha_{2}, \alpha_{3}$ ) or methylated and acetylated (for $\delta$ ) derivatives with respect to appropriate reference pigments. Protein was measured with the method of Lowry et al. (27) using bovine serum albumin as calibration standard.

\section{RESULTS}

Composition of bile pigments in bile and serum. Concentrations of biliverdin and bilirubins in gallbladder bile at various developmental stages are shown in Figure 1. Total bilirubin measured by HPLC accounted for $2.5-11.5 \%$ of total biliary bile pigment. Highest ratios of bilirubins to biliverdin were observed in embryonal bile. Values for total bilirubin in bile assayed by a conventional diazo-method were $2-7$-fold


\section{A GE (d a ys)}

Figure 1. Concentrations of biliverdin and bilirubins in bilc of chick embryos, chicks, and adult hens. Panel A shows the concentrations of total bilirubin, calculated by summation of the individual fractions: unesterified bilirubin, di-esterified bilirubin, and the two isomeric (C-8 and C-12) mono-esterified bilirubins. Panel $B$ depicts the biliverdin concentrations. Panel $C$ shows the total bilirubin/total bile pigment ratio. "Total bile pigment" refers to the sum of biliverdin and total bilirubin. 
higher than the total bilirubin concentrations assayed by HPLC. Why such large discrepancies occurred remains unresolved. Because the HPLC method is highly specific and sensitive, and is well validated analytically $(18,20)$, we believe that the HPLC results are accurate, and that the observed discrepancies reflect the presence in bile of diazo-positive material that does not correspond with authentic rubin pigment.

For all developmental stages tested, monomethyl esters and dimethyl esters of biliverdin-IX $\alpha$, -IX $\beta$, -IX $\gamma$, and -IX $\delta$ were undetectable in thin-layer chromatograms of pigment extracted from bile and subjected to alkaline methanolysis. A single biliverdin band migrating with the unesterified reference pigments biliverdin-IX $\alpha,-\mathrm{IX} \beta$, -IX $\gamma$, and IX $\delta$ (these four isomers were not separated by thin-layer chromatography) was observed in all chromatograms. Analysis of bile by HPLC was performed to determine the isomeric composition of this unesterified biliverdin band. Virtually all biliverdin corresponded with biliverdin-IX $\alpha$, with only trace amounts (less than $5 \%$ of total biliverdin) of the $\beta, \gamma$, and $\delta$ isomers.

Absence of significant amounts of bilirubin-IX $\beta$, -IX $\gamma$, or -IX $\delta$ was confirmed by thin-layer chromatography of the $O$ ethyl anthranilate azoderivatives prepared from bile. The socalled $\alpha_{\mathrm{F}}{ }^{1}, \alpha_{\mathrm{F}}, \beta \mathrm{x}$, and $\gamma$ fractions, which correspond with the azoderivatives from the non- $\alpha$ isomers of bilirubin-IX (19), were undetectable in these chromatograms.

Analysis of bile by alkaline methanolysis and normal-phase HPLC showed that the biliary bilirubins consisted almost exclusively of esterified pigments, with only a trace amount $(<2 \%$ of total rubins) of unconjugated bilirubin. Di-esterified bilirubin was predominant in all bile specimens including samples from 14- and 18-d-old embryos (Fig. 2A). Approximately one quarter of the esterified pigment was in monoesterified form, with a mean C-8/C-12 isomer ratio of 0.7 (Fig. $2 B$ ). The proportions of mono-esters to di-esters and of $\mathrm{C}-8$ isomer to $\mathrm{C}-12$ isomer tended to be higher in embryos.

Further characterization of the esterified bilirubins, particularly with respect to their conjugating moiety, was performed on $O$-ethyl anthranilate azo derivatives. Chromatography of azopyrromethenes extracted from bile after coupling with diazotized $O$-ethyl anthranilate demonstrated the presence of $\alpha_{2}$, $\alpha_{3}$, and $\delta$ azopigment fractions in all specimens, including those collected from 14- and 18-d-old embryos. The isolated azopigment fractions were acetylated (for $\alpha_{2}$, and $\alpha_{3}$ ) or methylated and then acetylated (for $\delta$ ), and the resulting derivatized azopyrromethenes were analyzed by thin-layer chromatography. Pigments obtained from $\alpha_{2}, \alpha_{3}$, and $\delta$ fractions migrated as correspondingly derivatized authentic Azpm(Xyl), Azpm(Glc), and Azpm(GlcU), respectively. Collectively, these findings confirmed that bilirubin glucosides, glucuronides, and xylosides were present in bile throughout development, with glucosides constituting $50-80 \%$ of total esterified bilirubin (Fig. 3).

The positional 1-, 2-, 3-, and 4-O-acyl isomers of bilirubin glucuronide in bile were also determined. The latter three non-glycosidic isomers are characteristically present in stagnant bile as seen in cholestasis (28). Separation of the four positional isomers by thin-layer chromatography after conversion of Azpm $(\mathrm{GlcU})$ to its methylated derivative demonstrated that at all developmental stages, biliary bilirubin glucuronides had almost exclusively $(>96 \%)$ the $1-O$-acyl isomeric structure.

Neither biliverdin nor esterified bilirubins were detectable in serum at any developmental stage. The detection limit of our HPLC assays was $0.4 \mu \mathrm{M}$ for biliverdin, $0.03 \mu \mathrm{M}$ for monoesterified bilirubins, and $0.06 \mu \mathrm{M}$ for di-esterified bilirubin (for a sample volume of $200 \mu \mathrm{l}$ serum). Similarly, unconjugated bilirubin was undetectable (i.e. $<0.4 \mu \mathrm{M}$ ) in serum from embryos, chicks, and adult chickens.

When serum was assayed by a standard diazo-method, a small amount of diazo-positivity was observed in some samples, giving calculated total bilirubin values ranging from 0 to $7 \mu \mathrm{M}$. In view of the undetectability of unconjugated or esterified bilirubins by the distinctly more sensitive and specific HPLC methods, it is unlikely that this diazo-positivity corresponded with rubin pigment.

Development of hepatic bilirubin UDP-glycosyltransferase activities. Bilirubin UDP-glycosyltransferase activities measured at various developmental stages with the cosubstrates UDPGlc, UDPGlcUA, and UDPXyl are shown in Figure 4. In agreement with the in vivo findings, esterification of bilirubin

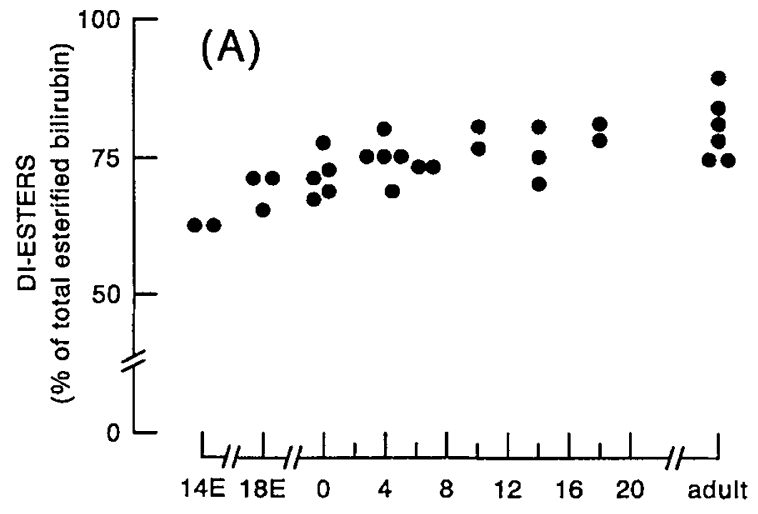

(B)

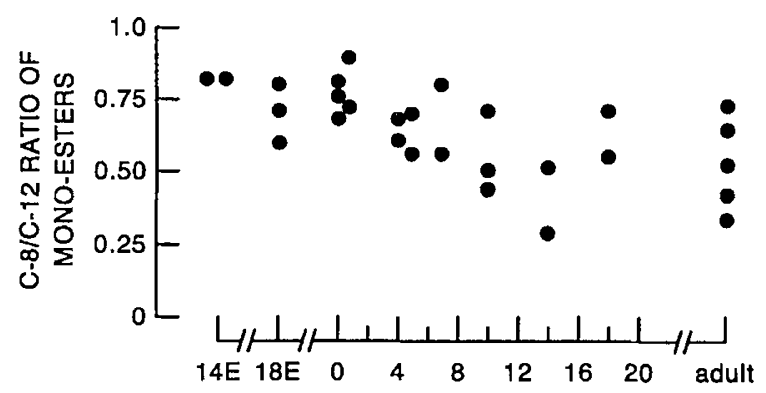

A GE (d a y s )

Figure 2. Composition of esterified bilirubins in bile during development. In panel A, di-esterified bilirubin is shown as a percentage of total esterified pigment. Panel $B$ gives the $\mathrm{C}-8 / \mathrm{C}-12$ isomer ratio of the mono-esterified bilirubin fraction. 


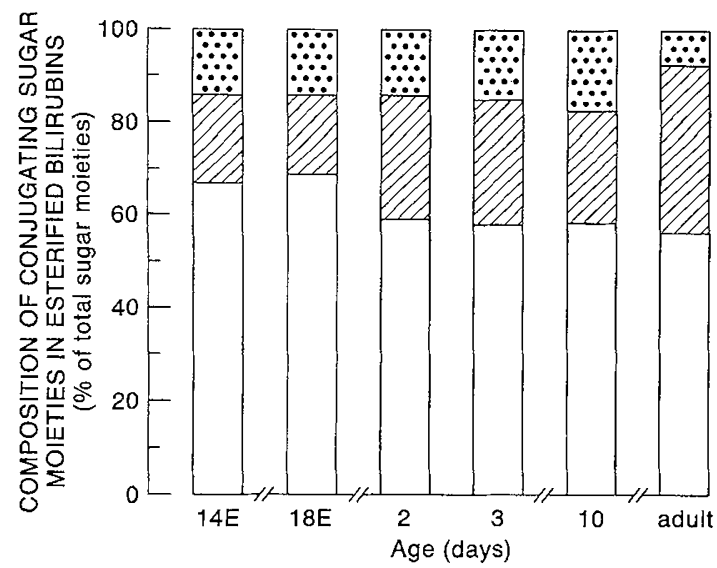

Figure 3. Composition of sugar moieties of esterified bilirubins in bile during development. The relative amounts (as percentages of total conjugating groups) of Glc, Xyl, and GlcU moieties are shown by, respectively, the open, dotted, and hatched parts of the bars.

was found in 14- and 18-d-old embryos and no major developmental changes occurred in any of the three measured UDP-glycosyltransferase-specific activities or in the ratio of any transferase activity over total conjugation capacity (Fig. 4). In 14- and 18-d-old embryos, specific activity of each transferase was comparable or only slightly lower than in adult animals (about 67,72 , and $102 \%$ of adult values, respectively, for bilirubin UDP-glucuronyl, UDP-glucosyl, and UDPxylosyltransferase). Highest specific activities were observed soon after hatching, between $\mathrm{d} 1$ and 2 , the lowest at $\mathrm{d} 14$.

Total conjugating capacity (sum of three enzymic activities), as shown in Figure $4 D$, averaged $84 \%$ of the adult value in $14-$ and 18-d-old embryos and peaked $1 \mathrm{~d}$ after hatching (79.9 \pm $15.9 \mathrm{pmol}$ of bilirubin $/ \mathrm{min} / \mathrm{mg}$ of protein). Among the three enzymic activities, UDP-xylosyltransferase and UDPglucuronyltransferase consistently accounted for, respectively, the highest $(41-50 \%)$ and lowest $(18-28 \%)$ contribution to total conjugating capacity throughout development (Fig. 4E).

\section{DISCUSSION}

The present study was made possible by the application of new and improved methods for analysis of bile pigments, which had not been available in the hitherto reported studies. These procedures included sensitive structural tests on esterified bilirubins and specific, sensitive HPLC methods for determination of bilirubins and biliverdins. Using these new experimental tools, we have demonstrated that hepatic bilirubin esterification is unquestionably present in the avian embryo. Quite unexpectedly, we found that no major changes in the in vivo and in vitro patterns of bilirubin esterification could be detected during the observed period of embryonic development and after hatching. At all stages, bile contained bilirubin glucuronides, xylosides, and predominantly glucosides. These in vivo results were confirmed in vitro by the demonstration of bilirubin UDP-glycosyltransferase activity in liver homogenates using the three corresponding UDP-sugar co-substrates, UDPGIcUA, UDPXyl, and UDPGlc.

A number of our findings require comment in light of the sometimes conflicting results published by others. The obser-
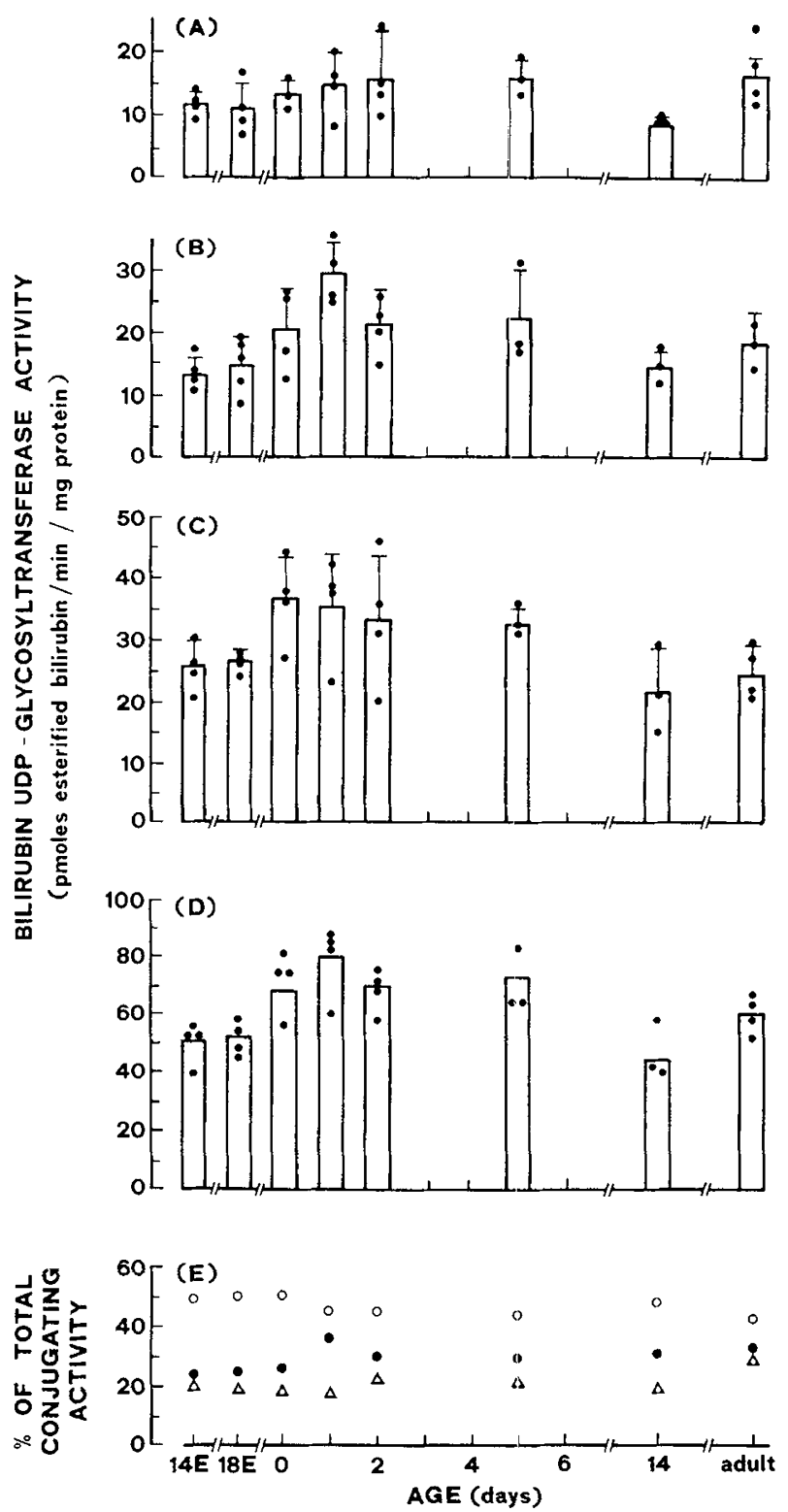

Figure 4. Development of hepatic bilirubin UDP-glycosyltransferase activities. Panels $A, B$, and $C$ show the transferase activities in liver homogenates toward, respectively, UDPGICUA, UDPGIc, and UDPXyl. The assays were performed on homogenates made from individual livers in the case of adult hens, and from pooled livers obtained from animals with identical age in the case of embryos and chicks. Panel $D$ shows total bilirubin-conjugating activity, i.e. the sum of the activities towards the three tested UDP-sugar cosubstrates. Results given are values from individual enzymic assays; the bars show means, with indication of $+1 \mathrm{SD}$ values. Panel $E$ depicts the contribution of each of the three bilirubin UDP-glycosyltransferase activities $(O$, with UDPXyl; , with UDPGIc; $\triangle$, with UDPGIcUA) to total conjugating activity.

vation that biliverdin amounts to over $90 \%$ of the biliary excreted bile pigment is in agreement with an earlier report (29). That unconjugated biliverdin is water soluble and can readily be excreted in bile most likely can be explained by the presence of a fixed methyne central bridge which makes it impossible for biliverdin to assume the conformational forms with multiple intramolecular hydrogen bonds as found in unconjugated bilirubin-IX $\alpha$ (30). The internal hydrogen bonds in unconjugated bilirubin-IX $\alpha$ effectively shield all polar groups 
of the pigment and fix the molecule in a rigid, ridge-tile shape, which may explain why unlike the unconjugated forms of biliverdin and bilirubin-IX $\beta$, IX $\delta$, and -IX $\gamma(31)$, unconjugated bilirubin-IX $\alpha$ is virtually insoluble in water and requires conjugation for effective biliary excretion. Our findings that neither biliverdin nor esterified bilirubins were detectable in serum at any developmental stage indicates that excretion of both types of pigment occurred very effectively, unlike excretion of unconjugated bilirubin-IX $\alpha$. We were unable to confirm the previously described hyperbilirubinemia and high biliary concentrations of bilirubins in the post-hatching period $(9,15)$. In fact, neither bilirubin nor biliverdin were detectable in serum at any developmental stage with highly sensitive and specific HPLC methods. A likely explanation for the apparent conflict between our findings and those of Sova et al. $(9,15)$ may be that diazo-positive compounds distinct from bilirubins are present in serum and bile of chicks and hens. For both bile and serum specimens we found large discrepancies between the results of the classical diazo-method and the values obtained with the highly specific HPLC method.

At least $95 \%$ of the bilirubin and biliverdin pigment excreted in avian bile was of the IX $\alpha$ isomeric type, with only trace amounts of the IX $\beta$, IX $\gamma$, and IX $\delta$ isomers at any developmental stage. This finding confirms the marked selectivity of the heme oxygenase-catalyzed cleavage of heme at the $\alpha$-meso bridge $(32,33)$, but differs from results reported by Blumenthal et al. (34) on human development. These authors found increased proportions of bilirubin-IX $\beta$ in gallbladder bile and intestinal contents of human embryos, and suggested that the embryonal, "immature" form(s) of heme oxygenase cleave heme less selectively at the $\alpha$-bridge than mature heme oxygenase. However, the isomeric composition of the bile pigments found in the stagnant bile and intestinal contents of these embryos may differ from the original pattern of the pigments formed by heme breakdown, because both the placental clearance mechanism and reabsorption from gallbladder and gut conceivably could cause selective removal of bilirubin-IX $\alpha$.

It is of interest to mention two lines of evidence indicating that good choleresis occurred throughout development of the chick embryos. First, we found no hyperbilirubinemia. Second, the sugar conjugates of bilirubin were present in bile exclusively in the 1-O-acyl isomeric form. This finding demonstrates that there was no bile stasis because the 1-O-acyl glycosides are rapidly transformed in stagnant bile to $2-O$-acyl, 3-O-acyl glycosides, and in the case of glucuronides also to the 4-O-acyl positional isomer (28).

Our findings on the relative amounts of bilirubin glucosides, xylosides, and glucuronides in bile of adult chickens are in agreement with previously published results $(11,35)$. Burchell et al. (10) previously reported that bilirubin glucuronyltransferase activity was undetectable in liver microsomes from 19-d chick embryos and 7-d hatched chicks. We were surprised, therefore, to observe that bilirubin glycosides were present in bile of chick embryos and chicks, with the same composition and at similar or even higher concentrations compared with adult bile. This demonstration of in vivo conjugation was fully corroborated by our in vitro results showing bilirubin UDPglycosyltransferase activity toward UDPGlcUA, UDPGIc, and
UDPXyl in liver homogenates at levels comparable to the adult values.

We speculate that the markedly different developments of hepatic bilirubin esterification in mammalian and avian species may reflect adaptive responses to the totally different conditions to which the mammalian and avian embryos are exposed. In the mammalian embryo, conversion of the water soluble nontoxic biliverdin, which cannot cross the placenta, to bilirubin seems useful because unconjugated bilirubin-IX $\alpha$ is easily transferred across the placenta $(36,37)$. If biliverdin were the end product of heme metabolism in the mammalian fetus, it would accumulate in the fetus. One might speculate that such accumulation could lead to product inhibition of heme oxygenase and impaired disposal of heme, which may be harmful. Detoxication of the potentially cytotoxic bilirubin occurs effectively by placental disposal, with availability of maternal mechanisms for final esterification and excretion. Under these conditions, lack of fetal bilirubin esterification seems advantageous because unlike the unconjugated pigment, esterified bilirubins cannot be transported effectively across the placenta $(36,37)$. Thus, it is only when the placental elimination mechanism disappears at birth that bilirubin esterification becomes useful and a necessity for self-detoxication. This interpretation is consistent with experimental evidence suggesting that birth-related rather than age-related factors are primary determinants of the development of hepatic bilirubin esterification in the mammalian fetus and newborn $(38,39)$. Unlike the mammalian fetus, the avian embryo cannot depend on a placenta and on maternal conjugation and excretion mechanisms for detoxication of unconjugated bilirubin. It seems logical therefore that the chick embryo develops its own detoxication system, by esterification of bilirubin, as soon as unconjugated bilirubin is formed from heme breakdown, which appears to occur at an early embryonal stage.

\section{REFERENCES}

1. Bonnett R 1978 Nomenclature. In: Dolphin D (ed) The Porphyrins. Academic Press, New York, Vol 1, pp 1-27

2. Dutton GJ 1980 Glucuronidation of Drugs and Other Compounds. CRC Press, Inc., Boca Raton, FL

3. Tephly TR, Burchell B 1990 UDP-glucuronosyltransferases: a family of detoxifying enzymes. Trends Pharmacol Sci 11:276-279

4. Miners JO, Mackenzie PI 1991 Drug glucuronidation in humans. Pharmacol Ther $51: 347-369$

5. Leakey JEA, Hume R, Burchell B 1987 Development of multiple activities of UDP-glucuronyltransferase in human liver. Biochem J 243:859-861

6. Dutton GJ 1978 Developmental aspects of drug conjugation, with special reference to glucuronidation. Annu Rev Pharmacol Toxicol 18:17-35

7. Greengard O, Thorndike J 1974 Changing enzyme patterns in chick organs during development. Enzyme 17:333-378

8. Dutton GJ 1973 Developmental studies on some enzymes associated with "detoxication". Enzyme 15:304-317

9. Sova Z, Koudela K, Matejusova D, Vrbenska A, Nemec Z 1971 Werte des GesamtBilirubins, freien Bilirubins, Bilirubin-Monoglukuronids und des BilirubinDiglukuronids in der Galle der Küken der weissen Leghorn-rasse während der frühen Postincubations-Ontogenese. Zenbralbl Veterinaermed Reihe A 18:835-841

10. Burchell B, Pratt GJ, Duffy I, West L 1983 Identification of increased amounts of UDP-giucuronyltransferase protein in phenobarbital-treated chick-embryo liver celis. Biochem J 214:517-523

11. Fevery J, Van de Vijver M, Michiels R, Heirwegh KPM 1977 Comparison in different species of biliary bilirubin-IX $\alpha$ conjugates with the activities of hepatic and renal bilirubin-IX $\alpha$-uridine diphosphate glycosyltransferases. Biochem J 164:737-746

12. Cornelius CE 1981 Hepatic bilirubin-IX $\alpha$-glycosyltransferase activities in animals excreting primarily biliverdin into bilc. Vet Clin Pathol 10:27-31

13. Tenhunen R 1965 Studies on bilirubin and its metabolism. II. Bilirubin in the bile of developing chick embryos and chicks. Ann Med Exp Biol Fenn 43:24-45

14. Leakey JEA, Wishart GJ, Dutton GJ 1976 Precocious development of UDPglucuronyltransferase activity in chick-embryo liver after administration of adreno- 
corticotropic hormone and of certain 11- $\beta$-hydroxy corticosteroids. Biochem $J$ 158:419-426

15. Sova Z, Holeckova L, Trefny D 1984 Hyperbilirubinemia in turkcys compared to that in goslings, ducklings, and Leghorn chicks during the first days after hatching. Sb Vys Sk Zemed Praze, Fak Agron Rada B B-40:9-19

16. Blanckaert N, Heirwegh KPM 1986 Analysis and preparation of bilirubins and biliverdins. In: Ostrow JD (ed) Bilirubin, Bile Pigments, and Jaundice. Marcel Dekker, New York, Chap 3, pp 31-79

17. Heirwegh KPM, Blanckaert N 1981 Analysis of bilirubin conjugates. Methods Enzymol 77:391-398

18. Blanckaert $N 1980$ Analysis of bilixubin and bilirubin mono- and di-conjugates. Determination of their relative amounts in biological samples. Biochem J 185:115128

19. Blanckaert N, Heirwegh KPM, Compernolle F 1976 Synthesis and separation by thin-layer chromatography of bilirubin-IX isomers. Their identification as tetrapyrroles and dipyrrolic ethyl anthranilate azo derivatives. Biochem J 155:405-417

20. Blanckaert N, Kabra PM, Farina FA, Stafford BE, Marton LJ, Schmid R 1980 Measurement of bilirubin and its monoconjugates and diconjugates in human serum by alkaline methanolysis and high-performance liquid chromatography. J Lab Clin Med 96:198-212

21. Blanckaert N, Fevery J, Heirwegh KPM, Compernolle F 1977 Characterization of the major diazo-positive pigments in bile of homozygous Gunn rats. Biochem $\ 164: 237$ 249

22. Novros JS, Thomas TR, Knoblock EC 1979 Improved method for accurate quantitation of total and conjugated bilirubin in serum. Clin Chem 25:1891-1899

23. Cole KD, Little GH 1982 Isocratic high-performance liquid chromatography of bile pigments. J Chromatogr 227:503-509

24. McDonagh AF 1979 Bile pigments: bilatriens and 5,15-Biliadienes. In: Dolphin D (ed) The Porphyrins. Academic Press, New York, Vol 6, pp 292-491

25. Vanstapel F, Blanckaert N 1987 On the binding of bilirubin and its structural analogs to hepatic microsomal bilirubin UDP-glucuronyltransferase. Biochemistry 26:60746082

26. Vanstapel F, Pua K, Blanckaert N 1986 Assay of mannose-6-phosphatase in untreated and detergent-disrupted rat liver microsomes for assessment of integrity of microsomal preparations. Eur J Biochem 156:73-77
27. Lowry OH, Rosebrough NJ, Farr AL, Randall RJ 1951 Protein measurement with the Folin phenol reagent. J Biol Chem 193:265-275

28. Blanckaert N, Compernolle F, Leroy P, Van Houtte R, Fevery J, Heirwegh KPM 1978 The fate of bilirubin-IX $\alpha$ glucuronide in cholestasis and during storage in vitro. Intramolecular rearrangement to positional isomers of glucuronic acid. Biochem J $171: 203-214$

29. Lin GL, Himes JA, Cornelius CE 1974 Bilirubin and biliverdin excretion by the chicken. Am J Physiol 226:881-885

30. Bonnett R, Davies JE, Hursthouse MB, Sheldrick GM 1978 The structure of bilirubin. Proc R Soc Lond B 202:249-268

31. Blanckaert N, Heirwegh KPM, Zaman Z 1977 Comparison of the biliary excretion of the four isomers of bilirubin-IX in Wistar and homozygous Gunn rats. Biochem J $164: 229-236$

32. Docherty JC, Masters BSS, Firneisz GD, Schacter BA 1982 Heme oxygenase provides $\alpha$-selectivity to physiological heme degradation. Biochem Biophys Res Commun 105:1005-1013

33. Bonkovsky HL, Healey JF, Pohl J 1990 Purification and characterization of heme oxygenase from chick liver. Comparison of the avian and mammalian enzymes. Eur J Biochem 189:155-166

34. Blumenthal SG, Stucker T, Rasmussen RD, Ikeda RM, Ruebner BH, Bergstrom DE, Hanson FW 1980 Changes in bilirubins in human prenatal development. Biochem J 186:693-700

35. Cornelius CE, Kelley KC, Himes JA 1975 Heterogeneity of bilirubin conjugates in several animal species. Cornell Vet 65:90-99

36. Schenker S, Dawber NH, Schmid R 1964 Bilirubin metabolism in the fetus. I Clin Invest 43:32-39

37. McDonagh AF, Palma LA, Schmid R 1981 Reduction of biliverdin and placental transfer of bilirubin and biliverdin in the pregnant guinea pig. Biochem J 194:273282

38. Campbell MT, Wishart GJ 1980 The effect of premature and delayed birth on the development of UDP-glucuronosyltransferase activities towards bilirubin, morphine and testosterone in the rat. Biochem J 186:617-619

39. Kawade N, Onishi S 1981 The prenatal and postnatal development of UDPglucuronyltransferase activity towards bilirubin and the effect of premature birth on this activity in the human liver. Biochem J 196:257--260 